\title{
Proteçấo à vida e à saúde da gravidez na adolescência sob o olhar da Bioética
}

I ${ }^{1}$ Caroline Filla Rosaneli, ${ }^{2}$ Natalia Bertani Costa, ${ }^{3}$ Viviane Maria Sutile I

Resumo: Este estudo foi realizado com o objetivo de analisar o perfil de adolescentes gestantes e de crianças nascidas de mães adolescentes no Estado do Paraná, identificando a proteção do direito à vida e à saúde sob o olhar da Bioética. Foi realizado um estudo epidemiológico quantitativo, utilizando-se indicadores da dimensão da saúde a partir de dados da plataforma digital de domínio público Cadê Paraná, em março e abril de 2016. Os dados foram investigados por meio de análise descritiva e da Bioética para um ensaio reflexivo. Detectaram-se 19.528 mães adolescentes e 21.580 filhos, 91,56\% encontravamse na faixa entre 15 a 17 anos, 58,52\% brancas e $82,67 \%$ residentes em domicílio urbano. A gravidez na adolescência é vista como situação de vulnerabilidade e risco social. Os números apresentados confrontaram os compromissos éticos e sociais. A intersetorialidade entre políticas sociais é fundamental para melhorar os indicadores. Esforços devem voltar-se para o contexto social e disparidades, a fim de garantir os direitos fundamentais da população estudada. Criar um ambiente favorável à igualdade de gênero, à saúde e aos direitos sexuais e reprodutivos dos adolescentes se faz prioridade entre ações e políticas públicas.

> Palavras-chave: gravidez; adolescência; direitos humanos; vulnerabilidade.

\author{
1 Programa de Pós-Graduação \\ em Bioética, Pontifícia \\ Universidade Católica do Paraná. \\ Curitiba-PR, Brasil (caroline. \\ rosaneli@gmail.com). \\ ORCID: 0000-0003-3710-5829 \\ ${ }^{2}$ Escola de Medicina, Pontifícia \\ Universidade Católica do Paraná \\ Curitiba-PR, Brasil (nataliabc36@ \\ hotmail.com). \\ ORCID: 0000-0003-4928-2830 \\ ${ }^{3}$ Programa de Pós-Graduação em \\ Bioética, Pontifícia Universidade \\ Católica do Paraná. Curitiba- \\ PR, Brasil (v.sutile@gmail.com). \\ ORCID: 0000-0003-1107-4729
}

Recebido em: 24/03/2019 Aprovado em: 23/07/2019 Revisado em: 07/02/2020 


\section{Introdução}

A gravidez na adolescência, juntamente com as mudanças fisiológicas e psicossociais naturais a este ciclo de vida, traz consigo riscos de morbidade e mortalidade, devido a fatores como gravidez precoce, aborto inseguro e doenças sexualmente transmissíveis.

A ausência ou ineficácia de abordagens estratégicas e açóes prioritárias sobre essas temáticas em populaçóes comumente negligenciadas são questôes que tornam a adolescente ainda mais vulnerável no que diz respeito ao direito à vida e à saúde (BEARINGER et al., 2007; BLUM et al., 2012; NUNES, 2012; SOUZA JUNIOR et al., 2018; GAUSMAN et al., 2019). No contexto da saúde para a infância e adolescência, é necessário considerar que a proteção do direito à vida e à saúde de crianças e jovens é dever social do Estado. Para tanto, informaçóes sobre a saúde sexual e reprodutiva aos jovens são um dilema em saúde pública, de fundamental diálogo e de políticas públicas eficazes.

A gravidez afeta eminentemente as trajetórias dessas vidas ao impulsionar as meninas à maternidade antes de estarem preparadas física, emocional ou financeiramente, por vezes perpetuando os ciclos intergeracionais de pobreza. Isso porque as meninas marginalizadas são frequentemente afetadas de forma desproporcional pela gravidez precoce. Contudo, a temática pode ser devastadora em todas as classes sociais, caso a parentalidade não seja planejada.

Em 2013, o Fundo das Naçóes Unidas sugere que a disponibilidade de contraceptivos modernos para adolescentes de 15 a 19 anos evitaria globalmente 2,1 milhóes de nascimentos não planejados, 3,2 milhões de abortos e 5.600 mortes maternas a cada ano (ONU, 2013). A maternidade nessa faixa etária é vista como uma situação de crise individual e risco social, uma vez que sua magnitude abrange questôes como abandono do estudo, conflitos familiares, discriminação social e alto risco de morbimortalidade materna e infantil (BUENDGENS; ZAMPIERI, 2012). É interessante salientar que $27 \%$ destas gestaçóes são planejadas pelas jovens brasileiras, e que existe uma associação entre este número e a baixa escolaridade (PRIETSCH et al., 2011). Mundialmente, o número de gravidezes planejadas pelas jovens varia de 9,11\% a 36\% (ALI; MOHAMMED; MUNGRUE, 2009).

O contexto e os determinantes da gravidez na adolescência variam entre os países; no entanto, a falta de conhecimento sobre sua saúde, direitos sexuais e reprodutivos, a 
educação e a renda limitada foram identificadas como fatores-chave que contribuem para a gravidez na adolescência na América Latina e Caribe (ONU, 2013).

O Brasil tem uma taxa de 65 gestaçóes para cada mil meninas, o que representa a sétima maior taxa de gravidez de adolescentes da América do Sul, segundo dados referentes ao período de 2006 a 2015. Entre estas, de cada cinco, três não trabalham nem estudam, sete em cada dez são afrodescendentes e aproximadamente a metade mora na Regiāo Nordeste do país (ONU, 2016). Entretanto, o Ministério da Saúde informa que o número absoluto de gestaçôes entre adolescentes brasileiras de 10 a 19 anos teve queda, após análise de relatório preliminar de dados do DATASUSSINASC 2017. A redução foi de 661.290 em 2004 para 546.529 em 2015 (BRASIL, 2017). Este número representa $18 \%$ dos 3 milhōes de nascidos vivos em 2015. A Região Sul está em quarto lugar, representando 11\%, ou 62.475 nascidos vivos. Atribui-se esta redução ao acesso a métodos contraceptivos pelas adolescentes em todas as unidades de saúde da rede pública do país, expansão do programa Saúde da Família e ações de educação sexual, como a Caderneta de Saúde do Adolescente (BRASIL, 2017; DAPES, 2009).

O percentual de mães com idades inferiores a 20 anos no Paraná, estado na Região Sul do Brasil, passou de 22,4\% em 2011 para 17,9\% em 2014, o que representa 1 a cada 6 nascidos vivos. Diante desta estatística, na maioria dos casos, as meninas passam a enfrentar problemas e a assumir responsabilidades para as quais não estão preparadas (SESI, 2017).

O Paraná é responsável por aproximadamente $9 \%$ do total de mães adolescentes brasileiras. A análise foi feita nos anos de 2008, 2009, 2011 e 2012, considerando números do Instituto Brasileiro de Geografia e Estatística. Em números absolutos lançados pelo relatório da plataforma CADÊ? de 2015, existiam 1.320 jovens na faixa etária de 12 a 14 anos e 12.776 de 15 a 17 anos até o ano de 2012. Estes dados colocam o Estado do Paraná na décima sétima posição em prevalência de gestação na adolescência na faixa etária de 12 a 14 anos e vigésima primeira posição entre 15 a 17 anos (MARISTA, 2015).

No processo de crescimento e desenvolvimento da criança que nasce de uma mãe adolescente, cuidados adequados poderão permitir seu pleno potencial de vida. Pessoas comprometidas com a garantia da vida dos vulneráveis devem integrar as práticas promotoras de saúde como forma de proteger a criança e atender o cumprimento dos direitos humanos elementares. 
Este estudo tem como objetivo analisar o perfil de adolescentes gestantes e de crianças nascidas de mães adolescentes no Estado do Paraná, na perspectiva da identificação de proteção do direito à vida e à saúde sob o olhar da bioética.

\section{Procedimentos metodológicos}

Trata-se de estudo epidemiológico quantitativo, que buscou dados através da plataforma digital de domínio público Cadê Paraná, ${ }^{1}$ que reúne informaçōes sobre a situação dos direitos humanos de meninos e meninas dos 399 municípios do Paraná (MARISTA, 2017). Com esta ferramenta é possível visualizar, comparar e analisar dados oficiais sobre oito dimensōes - saúde, educação, legislação, habitação, cidadania, demografia, economia e justiça, estabelecendo entre os dados o direito humano fundamental que aquela informação epidemiológica torna vulnerável ou desprotege os sujeitos. Neste trabalho foi utilizada a dimensão denominada Saúde.

Os dados foram coletados em março e abril de 2016, retirados de três indicadores da plataforma: 1) filhos tidos pelas mães de 10 a 17 anos, por situação de domicílio e cor ou raça, segundo a idade das mães; 2) mães de 10 a 17 anos de idade que tiveram filhos por situação do domicílio e cor ou raça, segundo a idade das mães; e 3) mães de 10 a 17 anos de idade que tiveram filhos, por nível de instrução, segundo a idade das mães.

Esses indicadores se relacionam ao direito fundamental à vida e à saúde. As variáveis utilizadas são dos anos de 2001 a 2016. Os dados foram investigados através de análise descritiva, por meio de avaliação da distribuição de frequência das variáveis quantitativas. Todos os dados coletados foram digitados no programa estatístico SPSS, versão 17.0. As reflexôes bioéticas para o ensaio reflexivo se deram a partir dos achados conjuntamente avaliados, baseando-se nos princípios fundamentais da Declaração Universal sobre Bioética e Direitos Humanos (UNESCO, 2005).

\section{Resultados}

Detectou-se no período analisado um total de 19.528 mães adolescentes, nos 399 municípios do Paraná, com a média de idade de 16 anos. O número total de filhos de mães adolescentes foi de 21.580, em média 1,10 filhos por mãe. Os demais dados estão resumidos nos quadros a seguir: 
Quadro 1. Distribuição por faixa etária de grávidas de 10 a 17 anos, de acordo com raça e domicílio no Paraná

\begin{tabular}{|l|c|c|c|c|c|c|c|c|c|c|c|}
\hline Idade & $\begin{array}{c}\text { Branca } \\
\text { Urbana }\end{array}$ & $\begin{array}{c}\text { Preta } \\
\text { Urbana }\end{array}$ & $\begin{array}{c}\text { Parda } \\
\text { Urbana }\end{array}$ & $\begin{array}{c}\text { Indígena } \\
\text { Urbana }\end{array}$ & $\begin{array}{c}\text { Branca } \\
\text { Rural }\end{array}$ & $\begin{array}{c}\text { Preta } \\
\text { Rural }\end{array}$ & $\begin{array}{c}\text { Parda } \\
\text { Rural }\end{array}$ & $\begin{array}{c}\text { Indígena } \\
\text { Rural }\end{array}$ & $\begin{array}{c}\text { Amarela } \\
\text { Urbana }\end{array}$ & $\begin{array}{c}\text { Amarela } \\
\text { Rural }\end{array}$ & Total \\
\hline $\mathbf{1 0}$ anos & 65 & 0 & 40 & 0 & 0 & 5 & 0 & 0 & 0 & 0 & $\mathbf{1 1 0}$ \\
\hline $\mathbf{1 1}$ anos & 79 & 10 & 21 & 0 & 14 & 0 & 6 & 10 & 0 & 0 & $\mathbf{1 4 0}$ \\
\hline $\mathbf{1 2}$ anos & 75 & 0 & 91 & 0 & 17 & 0 & 9 & 3 & 0 & 0 & $\mathbf{1 9 5}$ \\
\hline $\mathbf{1 3}$ anos & 104 & 0 & 119 & 10 & 12 & 14 & 23 & 2 & 0 & 0 & $\mathbf{2 8 4}$ \\
\hline $\mathbf{1 4}$ anos & 407 & 26 & 287 & 0 & 112 & 16 & 55 & 15 & 0 & 0 & $\mathbf{9 1 8}$ \\
\hline $\mathbf{1 5}$ anos & 1.181 & 72 & 747 & 0 & 260 & 0 & 200 & 41 & 35 & 0 & $\mathbf{2 . 5 3 6}$ \\
\hline $\mathbf{1 6}$ anos & 2.691 & 57 & 1.693 & 12 & 607 & 13 & 385 & 29 & 68 & 8 & $\mathbf{5 . 5 6 3}$ \\
\hline $\mathbf{1 7}$ anos & 4.903 & 274 & 3.037 & 8 & 902 & 30 & 519 & 70 & 33 & 6 & $\mathbf{9 . 7 8 2}$ \\
\hline Total & $\mathbf{9 . 5 0 5}$ & $\mathbf{4 3 9}$ & $\mathbf{6 . 0 3 5}$ & $\mathbf{3 0}$ & $\mathbf{1 . 9 2 4}$ & $\mathbf{7 8}$ & $\mathbf{1 . 1 9 7}$ & $\mathbf{1 7 0}$ & $\mathbf{1 3 6}$ & $\mathbf{1 4}$ & $\mathbf{1 9}$ \\
\hline
\end{tabular}

Fonte: as autoras.

Quadro 2. Distribuição de número de filhos nascidos no Paraná, nascidos de mães de 10 a 17 anos de idade, por situação de domicílio e cor ou raça

\begin{tabular}{|l|c|c|c|c|c|c|c|c|c|c|c|}
\hline Idade & $\begin{array}{c}\text { Branca } \\
\text { Urbana }\end{array}$ & $\begin{array}{c}\text { Preta } \\
\text { Urbana }\end{array}$ & $\begin{array}{c}\text { Parda } \\
\text { Urbana }\end{array}$ & $\begin{array}{c}\text { Indígena } \\
\text { Urbana }\end{array}$ & $\begin{array}{c}\text { Branca } \\
\text { Rural }\end{array}$ & $\begin{array}{c}\text { Preta } \\
\text { Rural }\end{array}$ & $\begin{array}{c}\text { Parda } \\
\text { Rural }\end{array}$ & $\begin{array}{c}\text { Indígena } \\
\text { Rural }\end{array}$ & $\begin{array}{c}\text { Amarela } \\
\text { Urbana }\end{array}$ & $\begin{array}{c}\text { Amarela } \\
\text { Rural }\end{array}$ & Total \\
\hline $\mathbf{1 0}$ anos & 65 & 0 & 40 & 0 & 0 & 5 & 0 & 0 & 0 & 0 & $\mathbf{1 1 0}$ \\
\hline $\mathbf{1 1}$ anos & 109 & 10 & 23 & 0 & 21 & 0 & 6 & 10 & 0 & 0 & $\mathbf{1 7 9}$ \\
\hline $\mathbf{1 2}$ anos & 96 & 0 & 132 & 0 & 34 & 0 & 9 & 3 & 0 & 0 & $\mathbf{2 7 4}$ \\
\hline $\mathbf{1 3}$ anos & 146 & 0 & 159 & 10 & 24 & 14 & 34 & 2 & 0 & 0 & $\mathbf{3 8 9}$ \\
\hline $\mathbf{1 4}$ anos & 451 & 26 & 303 & 0 & 127 & 16 & 80 & 15 & 0 & 0 & $\mathbf{1 . 0 1 8}$ \\
\hline $\mathbf{1 5}$ anos & 1.307 & 74 & 778 & 0 & 290 & 233 & 0 & 45 & 35 & 0 & $\mathbf{2 . 7 6 2}$ \\
\hline $\mathbf{1 6}$ anos & 2.951 & 57 & 1.847 & 12 & 638 & 13 & 429 & 29 & 84 & 8 & $\mathbf{6 . 0 6 8}$ \\
\hline $\mathbf{1 7}$ anos & 5.314 & 313 & 3.405 & 8 & 991 & 33 & 584 & 93 & 33 & 6 & $\mathbf{1 0 . 7 8 0}$ \\
\hline Total & $\mathbf{1 0 . 4 3 9}$ & $\mathbf{4 8 0}$ & $\mathbf{6 . 6 8 7}$ & $\mathbf{3 0}$ & $\mathbf{2 . 1 2 5}$ & $\mathbf{3 1 4}$ & $\mathbf{1 . 1 4 2}$ & $\mathbf{1 9 7}$ & $\mathbf{1 5 2}$ & $\mathbf{1 4}$ & 21.580 \\
\hline
\end{tabular}

Fonte: as autoras. 
Quadro 3. Distribuição por nível de instrução, segundo faixa etária das mães de 10 a 17 anos de idade que tiveram filhos no Paraná

\begin{tabular}{|l|c|c|c|c|c|}
\hline \multicolumn{1}{|c|}{ Idade } & $\begin{array}{c}\text { Não } \\
\text { determinado }\end{array}$ & $\begin{array}{c}\text { Sem instruçáo } \\
\text { e fundamental } \\
\text { incompleto }\end{array}$ & $\begin{array}{c}\text { Fundamental } \\
\text { completo e médio } \\
\text { incompleto }\end{array}$ & $\begin{array}{c}\text { Médio completo } \\
\text { e superior } \\
\text { incompleto }\end{array}$ & Total \\
\hline $\mathbf{1 0}$ anos & 0 & 110 & 0 & 0 & $\mathbf{1 1 0}$ \\
\hline $\mathbf{1 1}$ anos & 0 & 97 & 43 & 0 & $\mathbf{1 4 0}$ \\
\hline $\mathbf{1 2}$ anos & 0 & 180 & 15 & 0 & $\mathbf{1 9 5}$ \\
\hline $\mathbf{1 3}$ anos & 0 & 180 & 82 & 22 & $\mathbf{2 8 4}$ \\
\hline $\mathbf{1 4}$ anos & 14 & 748 & 133 & 22 & $\mathbf{9 1 7}$ \\
\hline $\mathbf{1 5}$ anos & 40 & 1.692 & 713 & 92 & $\mathbf{2 . 5 3 7}$ \\
\hline $\mathbf{1 6}$ anos & 41 & 3.408 & 2.008 & 106 & $\mathbf{5 . 5 6 3}$ \\
\hline $\mathbf{1 7}$ anos & 29 & 4.792 & 3.996 & 963 & $\mathbf{9 . 7 8 0}$ \\
\hline Total & 124 & 11.207 & 6.990 & 1.205 & 19.526 \\
\hline
\end{tabular}

Fonte: as autoras.

\section{Discussão}

O número absoluto de filhos nascidos de máes adolescentes de 10 a 17 anos de idade e o número absoluto de mães de 10 a 17 anos que tiveram filhos (21.580 filhos e 19.528 máes, respectivamente) pode ser considerado alto, sendo observado um viés esperado de escolaridade e situaçáo de domicílio, dado o contexto brasileiro de desenvolvimento, em que o estado do Paraná se insere (IBGE, 2012); 91,56\% das mães que tiveram filhos encontram-se na faixa de 15 a 17 anos; 90,87\% dos filhos são de mães nessa faixa etária. As mães de 17 anos correspondem a 50,09\% do total de mães analisadas; $58,52 \%$ do total das mães são brancas e $82,67 \%$ moram em domicílio urbano, estabelecendo assim o perfil das jovens paranaenses.

Deve-se destacar que 1.647 meninas abaixo de 14 anos se tornaram mães, e que, apesar de configurarem a minoria estatística, são a população em maior risco social. A gravidez destas meninas pode ter decorrido de flagrante violação dos direitos humanos, fruto de violência sexual e sexo por coação, além de representar um risco direto à vida, pois biologicamente há um despreparo físico para suportar as mudanças corporais advindas da gestação (NEAL et al., 2012). 
O Paraná se contrapóe ao perfil brasileiro no que se refere à autodenominação racial; no país a maioria das jovens mães é autodeclarada afrodescendente (ONU, 2016). Vale ressaltar que a divisão por cor ou raça não está de acordo com o preconizado pela Declaraçấo Universal sobre Bioética e Direitos Humanos, onde, em seu artigo $14^{\circ}$, referente à responsabilidade social e saúde, a distinção racial é rejeitada e a marginalização e a exclusão devem ser combatidas, para que a promoção da saúde e o desenvolvimento social sejam atingidos (UNESCO, 2005).

Em todas as faixas etárias, a baixa escolaridade foi um marco importante, informação que se assemelha ao restante do país. Idealmente, espera-se que uma jovem acima de 16 anos tenha completado o ensino fundamental e esteja cursando o ensino médio. Aproximadamente 61\% das jovens de 16 anos e 49\% na faixa etária de dos 17 não possuíam instrução ou tinham ensino fundamental incompleto. A cada ano adicional de estudo, o risco de engravidar durante a adolescência cai de 5 a 10\% (ONU, 2018). Um número maior de anos de estudo parece auxiliar numa melhor percepção em relação ao controle reprodutivo, métodos contraceptivos, além de maior exposição aos conceitos de educação sexual e conhecimento de consequências da gravidez na adolescência. Níveis educacionais mais altos se associam à possibilidade de uma maior reflexão, responsabilidade social e autonomia entre as adolescentes (ESTRADA et al., 2018; ONU, 2018; NUNES, 2012). O respeito à autonomia e a responsabilidade social são de grande importância dentro da bioética e ganham um artigo próprio $\left(5^{\circ}\right)$ na Declaração Universal sobre Bioética e Direitos Humanos (UNESCO, 2005).

Em seu artigo $22^{\circ}$, a garantia de educaçáo é mostrada como um papel dos Estados, e a educação por si só é considerada um instrumento promotor dos conceitos bioéticos (UNESCO, 2005). Além disso, a educação como fator de prevenção da gravidez é efetiva quando as meninas começam a estudar nos primeiros anos de vida, podem continuar a estudar por mais tempo, o espaço escolar é amigável e acessível, o Estado impulsiona oportunidades para o ensino superior, as jovens são protegidas contra exclusão, violência, expulsão do ambiente escolar, e o ensino é voltado ao conhecimento do próprio corpo, ao manejo da saúde reprodutiva, ao empoderamento nas relaçóes pessoais e aos direitos como cidadã (UNESCO, 2017).

Souza Junior e colaboradores (2018) descrevem os dilemas bioéticos na assistência médica às gestantes adolescentes numa revisão integrativa, denominando dilemas de ordem psicológica e biológica, sendo o aborto induzido um dos principais fatores 
de conflito. Trazem a bioética como portadora da reflexão e envolvimento dos profissionais de saúde em processos de educação e capacitaçáo, buscando proteger e garantir a plena realização dos direitos sexuais e reprodutivos das jovens mães.

A gravidez fruto de violência sexual é uma realidade vivenciada por jovens mães, principalmente em condições socioeconômicas mais baixas. $\mathrm{Na}$ intenção de compreender esta experiência emocional e física, o acolhimento institucional passa a ser uma alternativa. Miura et al. (2018) relatam que relaçôes familiares abusivas e negligentes, abandono e/ou violência do pai e do companheiro, uso de álcool e outras drogas pelos familiares e pelas adolescentes são histórias percebidas que favorecem rompimentos de vínculos e perpetuaçáo explícita da violência junto aos seus filhos. Neiva-Silva et al. (2018) demonstraram que, no caso de crianças e adolescentes sem vínculos com escola e com a família, os desfechos negativos à vida e à saúde são maiores ainda quando em situaçáo de rua. Entre os principais fatores de risco estão a gravidez e o aborto decorrentes do início precoce da vida sexual e a prática de sexo inseguro, que aumentam as vulnerabilidades dos meninos e meninas que vivem na rua.

A gravidez para os meninos e as meninas toma diferentes configuraçôes na vida, especialmente, na relação com a escola, o trabalho e na avaliação da própria gestação, conforme Bensaja dei Schiro e Koller (2013). Percebeu-se que a gravidez gerou mais sentimentos de vergonha nas meninas e indicação de necessidade de buscar trabalho para os meninos, e que na vida escolar há um reflexo de maior número de repetiçóes de ano entre meninos e de expulsóes ou desistência da escola em relação às meninas. A identificação dessas características de gênero e o reconhecimento das diferenças existentes para cada um dos pais são importantes para decisóes nas agendas de políticas públicas.

Almeida e Hardy (2007) analisaram a vulnerabilidade de gênero para a paternidade em homens adolescentes que incorporam precocemente papéis de trabalhador e pai, vivenciando a gravidez de sua parceira como a prova para sua condição de entrada para a vida adulta. Porém, o abandono parental por parte dos parceiros ou pais das crianças pode ser comum nestas situaçôes e, além de prejudicar a formação da pessoa humana, traz condiçôes jurídicas que merecem severa atuação do poder do Estado, segundo Lima e Duarte (2017).

Criar um ambiente favorável à igualdade de gênero, à saúde e aos direitos sexuais e reprodutivos dos adolescentes se faz prioridade entre açôes e políticas públicas, além de defesa dos direitos humanos desta população. 
Uma escolha consciente e informada para meninos e meninas sobre vida sexual e futuro os possibilita ter igualdade de oportunidades.

\section{Consideraçôes finais}

A necessidade de agir urgentemente para lidar com a gravidez na adolescência é consenso, empregando abordagens que cheguem às jovens mães e aos seus parceiros de forma apropriada à sua idade e capacidade de compreensão, adaptadas às necessidades locais, e focadas na equidade. Informaçôes de qualidade, acertadas e justas para adolescentes ajudam na tomada de decisão voluntária e responsável sobre sua saúde.

Ignorar políticas educacionais centradas na saúde sexual e reprodutiva é desproteger o futuro dessas jovens mães e das crianças que delas nascem. Garantir que uma menina sobreviva a todos os dilemas, contextos e enfrentamentos de uma gestação em idade precoce faz se primordial ao direito humano à vida dela e do bebê. ${ }^{2}$

\section{Agradecimentos}

À Rede Marista de Solidariedade e ao Centro Marista de Defesa da infância.

\section{Referências}

ALI, S.; MOHAMMED, S.; MUNGRUE, K. The epidemiology of unplanned pregnancies in North-Central Trinidad. Int J Adolesc Med Health, v. 21, n. 1, p. 73-77, 2009.

ALMEIDA, A. F. F.; HARDY, E. Vulnerabilidade de gênero para a paternidade em homens adolescentes. Rev. Saúde Pública, v. 41, n. 4, p. 565-572, 2007.

BEARINGER, L. H. et al. Perspectivas globais sobre a saúde sexual e reprodutiva de adolescentes: padrôes, prevenção e potencial. Lancet, v. 369, n. 9.568, p. 1220-1231, 2007.

BENSAJA DEI SCHIRO, D. E.; KOLLER, S. H. Ser adolescente e ser pai/mäe: gravidez adolescente em uma amostra brasileira. Estudos de psicologia, v. 18, n. 3, p. 447-455, 2013.

BLUM, R. W. et al. Adolescent health in the century XXI. Lancet, v. 379, n. 9826, p. 15671568, 2012.

BRASIL. Ministério da Saúde. DAPES. Planos de Açóes Estratégicas Gestão 2009-2010. Brasília, 19 de maio de 2009. Disponível em: <189.28.128.100/dab/docs.geral/plano_acoes_ estrategicas09_10telles.pdf>. Acesso em: $01 \mathrm{dez} 2017$.

- Ministério da Saúde. Portal da Saúde. Pesquisa Saúde Brasil. Brasília, DF, 2017. Disponível em: <http://portalsaude.saude.gov.br/indez.php/saudebrasil> Acesso em: 30 out 2017. 
BUENDGENS, B. B.; ZAMPIERI, M. F. M. A adolescente grávida na percepção de médicos e enfermeiros da atenção básica. Esc. Anna Nery, v. 16, n. 1, p. 64-72, 2012.

ESTRADA, L. F. et al. Factors associated with desire pregnancy among adolescent women in five Latin American countries: a multilevel analysis. BJOG, v. 125, n. 1, p. 1330-1336, 2018.

GAUSMAN, J. et al. How do Jordanian and Syrian youth living in Jordan envision their sexual and reproductive health needs? A concept mapping study protocol. BMJ Open, n. 9, p. e027266, 2019.Doi: 10.1136 / bmjopen-2018-027266.

INSTITUTO BRASILEIRO DE GEOGRAFIA E ESTATÍSTICA. Sintese de indicadores sociais 2012: uma análise das condiçôes de vida da população brasileira. Coordenação de População e Indicadores Sociais. Rio de Janeiro, 2012.

LIMA, G. D. S.; DUARTE, H. G. Os reflexos do abandono parental e a plena consecução do fundamento do nosso Estado dignidade da pessoa humana. Âmbito Jurídico, Rio Grande, v. XX, n. 156, jan 2017. Disponível em: <http://www.ambito-juridico.com.br/site/index.php?n_ link=revista_artigos_leitura\&artigo_id=18396\&revista_caderno=14 >. Acesso em: fev 2019.

MARISTA. Portal CADÊ? Brasil. Cadê 2015 - Crianças e Adolescentes em Dados Estatísticos. Disponível em: <http://marista.edu.br/social/wp-content/uploads/2016/05/Relat\%C3\%B3rioCADE-2015-vers\%C3\%A3o-final_site.pdf>. Acesso em: 30 nov 2017.

MIURA, P. O.; TARDIVO, L. S. L. C; BARRIENTOS, D. M. S. O desamparo vivenciado por mães adolescentes e adolescentes grávidas acolhidas institucionalmente. Ciênc. saúde coletiva, v. 23, n. 5, p. 1601-1610, 2018.

NEAL, S. M.; et al. Childbearing in adolescents aged 12-15 years in low resource countries: a neglected issue. New estimates from demographic and household surveys in 42 countries. Acta Obstet Gynecol Scand; v. 91, p. 1114-18, 2012.

NEIVA-SILVA, L. et al. Experiência de gravidez e aborto em crianças, adolescentes e jovens em situação de rua. Ciência \& Saúde Coletiva, v. 23, n. 4, p. 1055-1066, 2018.

NUNES, S. A. Esperando o futuro: uma maternidade na adolescência. Physis: Revista de Saúde Coletiva. Rio de Janeiro, v. 22, n.1, p. 53-75, 2012.

ORGANIZAÇÃO DAS NAÇÓES UNIDAS (ONU). Fundo de População das Nações Unidas (UNFPA). A situação da população mundial 2018: o poder de escolha - direitos reprodutivos e a transição demográfica. Brasília, 2018. Disponível em: https://brazil.unfpa.org/sites/default/ files/pub-pdf/SWOP_2018.pdf. Acesso em: 1 fev 2019.

. Fundo de População das Nações Unidas (UNFPA). O estado da população mundial 2013: a maternidade na infância. Nova York, 2013. Disponivel em: http://www.unfpa.org/ publications/state-world-population-2013. Acesso em: 1 fev 2019. 
. Fundo de População das Nações Unidas (UNFPA). State of World Population 2016. Disponível em: <http://www.unfpa.org/swop-2016>. Acesso em: 7 dez 2018.

. Sistema de las Naciones Unidas en El Salvador. Situación y desafíos de la juventud en Iberoamérica. San Salvador: ONU, 2008.

ORGANIZAÇÃO MUNDIAL DA SAÚDE. Diretrizes da OMS sobre prevenção da gravidez precoce e resultados reprodutivos ruins entre adolescentes em paises em desenvolvimento. Genebra: OMS, 2011. Disponível em: http://www.who.int/maternal_child_adolescent/documents/ preventing_early_pregnancy/en/. Acesso em: 07 dez 2018.

PRIETSCH, S. O. M. et al. Gravidez não planejada no extremo Sul do Brasil: prevalência e fatores associados. Caderno Saúde Pública, v .27, n. 10, p. 1906-1916, 2011.

SERVIÇO SOCIAL DA INDÚSTRIA (SESI). Portal ODM. Relatórios dinâmicos. Disponível em: <www.relatoriosdinamicos.com.br/portalodm/>. Acesso em: 15 nov 2017.

SOUZA JUNIOR, E. V. et al. Dilemas bioéticos na assistência médica às gestantes adolescentes. Rev. Bioética, v. 26, n. 1, p. 87-94, 2018.

UNESCO. Declaração Universal sobre Bioética e Direitos Humanos. 2005. Disponível em: <http://unesdoc.unesco.org/images/0014/001461/146180por.pdf>. Acesso em: 05 nov 2017.

. Early and unintended pregnancy o the education sector: Evidence review and recommendations. Paris: UNESCO, 2017. 68 p.

\section{Notas}

${ }^{1}$ (http://www.cadeparana.org.br/_dimensoes/saude)

${ }^{2}$ C. F. Rosaneli participou da concepção do projeto, coleta e interpretação dos dados, na redação e revisão final do artigo. N. B. Costa participou da coleta e interpretaçáo dos dados, e na redaçáo final do artigo. V. M. Sutile contribuiu para a análise dos dados, redação e revisão do artigo. 


\section{Abstract}

Protection of the life and health of teenage
pregnancy from the perspective of Bioethics This study aimed to analyze the profile of pregnant teenagers and children born to teenage mothers in the State of Paraná, Brazil, identifying the protection of the right to life and health from the perspective of Bioethics. A quantitative epidemiological study was carried out, using health dimension indicators from data from the public domain digital platform Cadê Paraná, in March and April 2016. The data were investigated through descriptive analysis and Bioethics for an essay reflective; 19,528 teenage mothers and 21,580 children were detected, $91.56 \%$ were between 15 and 17 years old, $58.52 \%$ were white and $82.67 \%$ lived in urban households. Adolescent pregnancy is seen as a situation of vulnerability and social risk. The figures presented confronted ethical and social commitments. The intersectorality between social policies is essential to improve the indicators. Efforts must focus on the social context and disparities in order to guarantee the fundamental rights of the population studied. Creating an environment favorable to gender equality, health and sexual and reproductive rights of adolescents is a priority among public actions and policies.

> Keywords: pregnancy; adolescence; human rights; vulnerability. 